\title{
X-ray reflected spectra from accretion disk models. II. Diagnostic tools for X-ray observations
}

\author{
J. García ${ }^{1,2}$, T.R. Kallman², \\ and \\ R.F. Mushotzky ${ }^{3}$
}

Received —

\footnotetext{
${ }^{1}$ Department of Physics, Western Michigan University, Kalamazoo, MI 49008, USA javier.garcia@wmich.edu
}

${ }^{2}$ NASA Goddard Space Flight Center, Greenbelt, MD 20771 timothy.r.kallman@nasa.gov

${ }^{3}$ Department of Astronomy, University of Maryland, College Park, MD, USA richard@astro.umd.edu 


\begin{abstract}
We present a comprehensive study of the emission spectra from accreting sources. We use our new reflection code to compute the reflected spectra from an accretion disk illuminated by X-rays. This set of models covers different values of ionization parameter, solar iron abundance and photon index for the illuminating spectrum. These models also include the most complete and recent atomic data for the inner-shell of the iron and oxygen isonuclear sequences. We concentrate our analysis to the $2-10 \mathrm{keV}$ energy region, and in particular to the iron K-shell emission lines. We show the dependency of the equivalent width (EW) of the Fe $\mathrm{K} \alpha$ with the ionization parameter. The maximum value of the $\mathrm{EW}$ is $\sim 800 \mathrm{eV}$ for models with $\log \xi \sim 1.5$, and decreases monotonically as $\xi$ increases. For lower values of $\xi$ the $\mathrm{Fe} \mathrm{K} \alpha \mathrm{EW}$ decreases to a minimum near $\log \xi \sim 0.8$. We produce simulated CCD observations based on our reflection models. For low ionized, reflection dominated cases, the $2-10 \mathrm{keV}$ energy region shows a very broad, curving continuum that cannot be represented by a simple power-law. We show that in addition to the Fe K-shell emission, there are other prominent features such as the Si and S L $\alpha$ lines, a blend of Ar VIII-XI lines, and the Ca X $\mathrm{K} \alpha$ line. In some cases the $\mathrm{S}$ Xv blends with the He-like Si RRC producing a broad feature that cannot be reproduced by a simple Gaussian profile. This could be used as a signature of reflection.
\end{abstract}

\title{
1. Introduction
}

Accreting systems are observed to emit copious radiation in the X-ray energy range which suggests emission from the innermost regions of an accretion disk. Analysis of the 
X-ray spectra is crucial to study the complex mixture of emitting and absorbing components in the circumnuclear regions of these systems. However, there are only few observables that provide indication of the existence of an accretion disk. These need to be understood in order to correctly interpret the physics of these systems. In a general sense, the observed radiation from accreting sources can be divided into: a thermal component, in the form of a black body emitted at the surface of the disk with typical temperatures of $\sim 0.01-10 \mathrm{keV}$ (for the mass range $\sim 10^{8}-10 M_{\odot}$ ); a coronal component in the form of a power law covering energies up to $\sim 100 \mathrm{keV}$, believed to arise from inverse Compton scattering in a hot gas that lies above the disk; and a reflected component, resulting from the interaction of some of the coronal X-ray photons and the optically thick material of the disk. In the reflected component, the most prominent feature is the iron $\mathrm{K} \alpha$ emission line at $\sim 6.4 \mathrm{keV}$, produced by transitions of electrons between the $1 s$ and $2 p$ atomic orbitals. These are ubiquitous in the spectra of accreting sources (Pounds et al. 1990; Nandra \& Pounds 1994; Miller 2007). Other reflection signatures are the so called Compton shoulder (next to the Fe K-line), and the Compton hump (above $\sim 10 \mathrm{keV}$ ), produced by the down-scattering of high energy photons by cold electrons.

Much theoretical effort has gone into studies of X-ray illuminated disks over the past few decades. Most models assume that the gas density is constant with depth (Done et al. 1992; Ross \& Fabian 1993; Matt et al. 1993; Czernv \& Zvcki 1994; Krolik et al. 1994; Magdziarz \& Zdziarski 1995; Ross et al. 1996; Matt et al. 1996; Poutanen et al. 1996; Blackman 1999). Although constant density models may be appropriate for radiation-pressure dominated disks, other studies have shown significant differences when the gas density is properly solved via hydrostatic equilibrium (Rozanska \& Czernv 1996; Navakshin et al. 2000; Navakshin \& Kallman 2001; Ballantyne et al. 2001; Dumont et al. 2002; Ross \& Fabian 2007). Recently we have developed a new model for the reflected spectra from illuminated accretion disks called XILLVER (García \& Kallman 2010). Although 
our code is similar in its principal assumptions to previous models, XILLVER includes the most recent and complete atomic data for K-shell of all relevant ions (Kallman et al. 2004; García et al. 2005; Palmeri et al. 2008; García et al. 2009; Palmeri et al. 2010). This has a dramatic impact on the predicted spectra, in particular the $\mathrm{K} \alpha$ emission from iron. With this model we can study the effects of incident X-rays on the surface of the accretion disk by solving simultaneously the equations of radiative transfer and ionization equilibrium over a large range of column densities. Plane-parallel geometry and azimuthal symmetry are assumed, such that each calculation corresponds to an annular ring at a given distance from the source of X-rays. The redistribution of photons due to Compton down-scattering is included by using a Gaussian approximation for the Compton kernel. With XILLVER we are able to solve the reflection problem with great detail, i.e., with very high energy, spatial and angular resolution.

In this paper we present a systematic analysis of our models for reflected spectra from X-ray illuminated accretion disks. We show how the most relevant atomic features in the spectra depend on the assumed properties of the irradiated gas. We pay particular attention to the Fe K-shell emission lines, and we quantify its strength in terms of the equivalent widths predicted by our models. These models are also used to produce faked CCD spectra in order to simulate the effects of the instrumental response and limited spectral resolution. These results will be helpful diagnostic tools in the interpretation of accreting sources observations.

In the next Section we describe briefly the numerical methods used in our reflection code. In Section 3 we present the results of our analysis on the simulated spectra, as well as comparisons with observations from Seyfert galaxies and X-ray binaries. The main conclusions are presented in Section 4 


\section{The Reflection Model}

In order to calculate the reflected spectra from X-ray illuminated accretion disks we make use of our reflection code XILLVER. The details of the calculations are fully described in García \& Kallman (2010), thus here we just review the main aspects.

The description of the interaction of the radiation with the gas in the illuminated slab requires the solution of the transfer equation:

$$
\mu^{2} \omega^{2}(E, \tau) \frac{\partial^{2} u(\mu, E, \tau)}{\partial \tau^{2}}+\mu^{2} \omega(E, \tau) \frac{\partial \omega(E, \tau)}{\partial \tau} \frac{\partial u(\mu, E, \tau)}{\partial \tau}=u(\mu, E, \tau)-S(E, \tau)
$$

where $u(\mu, E, \tau)$ is the average intensity of the radiation field for a given cosine of the angle with respect to the normal $\mu$, energy $E$, and position in the slab, specified by the Thomson optical depth $d \tau \equiv-\alpha_{\mathrm{T}} d z=-\sigma_{T} n_{e} d z$, where $\sigma_{T}$ is the Thomson cross section $\left(=6.65 \times 10^{-25} \mathrm{~cm}^{2}\right)$, and $n_{e}$ is the electron number density. The quantity $\omega$ is defined

as the ratio of the Thomson scattering coefficient $\alpha_{\mathrm{T}}$ to the total opacity $\chi(\tau, E)$. The second term in the right-hand side of Equation 1 is the source function, which is defined as the ratio of the total emissivity over the total opacity of the gas, taking into account both scattering and absorption processes:

$$
S(E, \tau)=\frac{\alpha_{k n}(E)}{\chi(E, \tau)} J_{c}(E, \tau)+\frac{j(E, \tau)}{\chi(E, \tau)}
$$

where $\alpha_{k n}(E)$ is the Klein-Nishina scattering coefficient, $j(E, \tau)$ is the thermal continuum plus lines emissivity, and $J_{c}(E, \tau)$ is the Comptonized mean intensity, given by the Gaussian convolution

$$
J_{c}(E, \tau)=\frac{1}{\sigma \pi^{1 / 2}} \int_{0}^{\infty} d E^{\prime} \exp \left[\frac{-\left(E-E_{c}\right)^{2}}{\sigma^{2}}\right] \int_{0}^{1} u\left(\mu, E^{\prime}, \tau\right) d \mu
$$

The Gaussian is centered at $E_{c}=E^{\prime}\left(1+4 \theta-E^{\prime} / m_{e} c^{2}\right)$, where $m_{e}$ is the electron mass, $c$ is the speed of light, and $\theta=k T / m_{e} c^{2}$ is the dimensionless temperature. The energy dispersion is given by $\sigma=E^{\prime}\left[2 \theta+\frac{2}{5}\left(E^{\prime} / m_{e} c^{2}\right)^{2}\right]^{1 / 2}$. 
The solution of the system is completed by imposing two boundary conditions. At the top of the slab $(\tau=0)$, we specify the incoming radiation field incident at a given angle $\mu_{0}$ by

$$
\omega(0, E) \mu\left[\frac{\partial u(\tau, \mu, E)}{\partial \tau}\right]_{0}-u(0, \mu, E)=-\frac{2 F_{\mathbf{x}}(E)}{\mu_{0}} \delta\left(\mu-\mu_{0}\right)
$$

where $F_{\mathrm{x}}(E)$ is the net flux of the illuminating radiation incident at the top of the slab. At the inner boundary $\left(\tau=\tau_{\max }\right)$, we specify the outgoing radiation field to be equal to a blackbody with the expected temperature for the disk:

$$
\omega\left(\tau_{\max }, \mu, E\right) \mu\left[\frac{\partial u(\tau, \mu, E)}{\partial \tau}\right]_{\tau_{\max }}+u\left(\tau_{\max }, \mu, E\right)=B\left(T_{\text {disk }}\right)
$$

where $T_{\text {disk }}$ can be defined using the Shakura \& Sunvaev (1973) formulae. Since these models are calculated under the assumption of constant density, we use the common definition of the ionization parameter (Tarter et al. 1969) to characterize each case:

$$
\xi=\frac{4 \pi F_{\mathrm{x}}}{n_{e}} .
$$

where $n_{e}$ is the electron gas density, and $F_{\mathrm{x}}$ is the net illuminating flux integrated in the $1-1000$ Ry energy range. The solution of the system is found by forward elimination and back substitution. A full transfer solution must be achieved iteratively in order to self-consistently treat the scattering process. This procedure requires $\sim \tau_{\max }^{2}$ iterations (lambda iterations) for convergence.

Given the solution for the radiation field at each point in the atmosphere, we use the photoionization code XSTAR (Kallman \& Bautista 2001) to determine the state of the gas at each point of the gas. The state of the gas is defined by its temperature and the level populations of the ions. The relative abundances of the ions of a given element and the level populations are found by solving the ionization equilibrium equations under the assumption of local balance, subject to the constraint of particle number conservation for each element. XSTAR calculates level populations, temperature, the opacity $\chi(E, \tau)$ and the 
total emissivity $j(E, \tau)$ of the gas assuming that all the physical processes are in steady state and imposing radiative equilibrium.

The XSTAR atomic database collects recent data from many sources including CHIANTI (Landi \& Phillips 2006), ADAS (Summers 2004), NIST (Ralchenko et al. 2008), TOPbase (Cunto et al. 1993) and the IRON project (Hummer et al. 1993). The database is described in detail by Bautista \& Kallman (2001). Additionally, the atomic data associated with the K-shell of the Fe ions incorporated in the current version of XSTAR has been recently calculated and represents the most accurate and complete set available to the present. A compilation of these results and a careful study of their impact on the photoionization models can be found in Kallman et al. (2004). Moreover, XSTAR also includes the atomic data relevant to the photoabsorption near the K edge of all oxygen (García et al. 2005), and nitrogen ions (García et al. 2009).

Finally, all the calculations presented in this paper were carried out over a large optical depth $\left(\tau_{\max }=10\right)$, considering high resolution spectra with an energy grid of at least $5 \times 10^{3}$ points $(\mathcal{R}=E / \Delta E \sim 350), 200$ spatial zones, and 50 angles to account for anisotropy of the radiation field. The simulations do not take into account the dynamics of the system. Input parameters common to all these models are: the electron gas density $n_{e}=10^{15} \mathrm{~cm}^{-3}$, photon index of the incident radiation $\Gamma=2$, mass of the central object $M=10^{8} M_{\odot}$, distance from the central object $R=7 R_{s}$, and the mass accretion rate $\dot{M}=1.6 \times 10^{-3} \dot{M}_{E d d}$, where $R_{s}=2 G M / c^{2}$ is the Schwarzschild radius and $\dot{M}_{E d d}$ is the accretion rate at the Eddington limit. This particular set of parameters yields a disk effective temperature of $T_{\text {disk }}=2.8 \times 10^{4}{ }^{\circ} \mathrm{K}$. The models presented here cover 10 different values for the illumination flux $F_{\mathrm{x}}=5 \times 10^{14}-5 \times 10^{17} \mathrm{erg} \mathrm{cm}^{-2} \mathrm{~s}^{-1}$, which corresponds to ionization parameters of $\log \xi=0.8,1.1,1.5,1.8,2.1, \ldots, 3.8$. It is important to notice that these simulations are not constrained to a particular geometry for the illumination. This is because the flux $F_{\mathrm{x}}$ of the 
illuminating radiation is defined at the surface of the slab, regardless of the position of the X-ray source. Therefore, a set of models with different values of the ionization parameter can be used to construct a particular geometry, by defining the luminosity and location of the source. We do not consider models with ionization parameters lower than $\sim 4 \pi$ $(\log \xi=0.8)$, since our code is optimized for calculations of medium to high ionization. The upper limit is set to $\log \xi=3.8$, because at such a high illumination the gas is almost completely ionized over a large depth.

\section{Results}

\subsection{Equivalent Widths}

The equivalent width of a line provides a quantitative measure of the strength of the spectral profile, both in emission or absorption. It is defined by the well known formula

$$
E W=\int_{E_{\text {low }}}^{E_{\text {high }}} \frac{\left(F(E)-F_{c}(E)\right)}{F_{c}(E)} d E,
$$

where $F(E)$ is the total flux and $F_{c}(E)$ is the total flux of the continuum under the line. The integration is performed over the energy range where the spectral feature takes place, between $E_{\text {low }}$ and $E_{\text {high }}$. Usually, there are uncertainties in the determination of the intrinsic continuum which affects the knowledge of the integration region and the actual value of the equivalent width itself. Nonetheless, one can approximate its calculation by defining an energy region in which one knows only the spectral feature of interest appears, and where a local continuum can be defined.

In this paper we make use of the equivalent width as a measure of the strength of several features in the X-ray spectra reflected from illuminated accretion disk. As an example, in Figure 1 we show the reflected spectra from 3 different models calculated for $\log \xi=0.8,1.8$ and 2.8 in the $4-9 \mathrm{keV}$ energy region, where the only atomic features are 
due to inner-shell transitions from Fe ions. The spectra (flux vs. energy) are shown as solid lines. Vertical dotted lines are placed at $5.5 \mathrm{keV}$ and $7 \mathrm{keV}$, defining a particular integration region. The continuum is defined as a straight line that passes through these two points in the spectra, which is shown as dashed lines. It is clear from the Figure that the resulting continuum does a good job reproducing the local continuum and that it is not superimposed over any part of the emission profile. The upper limit $E_{h i g h}=7 \mathrm{keV}$ was chosen such that only emission from K $\alpha$ transitions of Fe are taken into account, neglecting any $\mathrm{K} \beta$ emission (which occurs at energies above $7 \mathrm{keV}$ ). The lower limit, however, is more arbitrary. It needs to be chosen such that all the line profile is included in the integration. If $E_{\text {low }}$ is set too large, part of the line emission can fall outside the range, especially for cases with large values of the ionization parameter, where Comptonization smears the line profile due to the down-scattering. However, if $E_{l o w}$ is set to a small value, all the line emission is taken into account but the local continuum is modified to a point that could either under- or over-estimate the strength of the line. Therefore, we have repeated the calculation of the equivalent widths for $E_{l o w}=5,5.5$ and $6 \mathrm{keV}$, in order to evaluate the sensitivity of the results on this lower limit.

Figure 2 shows the resulting equivalent widths as a function of the ionization parameter, calculated for the Fe K $\alpha$ emission profile in 10 of our models. Circles connected with solid lines correspond to the integration range 5-7 keV, squares connected with dashed lines to 5.5-7 keV, and triangles connected with short-dashed lines to the integration performed over the 6-7 keV energy range. It is clear from the Figure that the equivalent widths are almost unaffected by the variations of the lower boundary of the integration range, with the exception of those models with $1.5<\log \xi<2.5$. These are the most sensitive cases probably because of the complexity of the iron $\mathrm{K}$ emission. For lower values of the ionization parameter, the emission mainly occurs at $6.4 \mathrm{keV}$ due to mostly neutral Fe ions. Higher values of $\xi$ means that the gas is highly ionized and thus mostly He- and H-like Fe ions are 
responsible for the emission at $\sim 6.9 \mathrm{keV}$. In both cases the line profile is simple in the sense that the emission is concentrated at one particular energy. In between, emission from many different ions takes place at the same time, creating a more complex spectral feature, as can be seen in the spectrum for $\log \xi=1.8$ in Figure 1, Since there is no clear reason to choose one of these values of $E_{\text {low }}$, we choose the intermediate one $\left(E_{\text {low }}=5.5 \mathrm{keV}\right)$. We consider the uncertainties in the equivalent widths to be of order of the differences between the values shown in Figure $2(\leq 100 \mathrm{eV})$.

Figure 3 shows a comparison of the Fe $\mathrm{K} \alpha$ equivalent widths predicted by our models (circles connected with dashed lines), and those predicted by the models included in REFLION (triangles connected with solid lines, Ross \& Fabian 2005). The calculation of the equivalent widths is the same in both models, with the integration performed in the 5.5-7 keV energy range. There are important differences to notice in this comparison. It is convenient to make the distinction between two regions in the plot; the region for models with $\log \xi>1.5$ and the one for models with $\log \xi<1.5$. In the high ionization region, both sets of models show a similar behavior, the equivalent widths decrease monotonically as the ionization parameter increases, resembling the Baldwin effect for X-rays. Nevertheless, in this region all our models systematically predict stronger $\mathrm{Fe} \mathrm{K} \alpha$ emission. For those models in the low ionization region $(\log \xi<1.5)$, the differences are significantly larger. In fact, REFLION models predict that the equivalent widths keep growing as the ionization parameter decreases, while our models show the opposite trend. This turn over in the values of the equivalent widths can be understood by looking the ionization balance in detail. Kallman et al. (2004) performed similar calculations in photoionized models using the same atomic data used in our models. Using a set of XSTAR models of thin spherical shells illuminated by the same power law spectrum they calculated the ratio of the emissivity per particle for $\mathrm{K}$ line production as a function of the ionization parameter. Their Figure 7 shows the contribution of each ion of iron summing over the $\mathrm{K}$ lines for each one. The overall 
behavior of these models is quite similar to the ones presented in this paper. The combined line emissivities have a minimum just below $\log \xi \sim 1$ (roughly where our calculations start), and is mainly due to a combination of Fe XIV-XVI. As the ionization increases the also the emissivities increase, with maximum values that peak around $\log \xi \sim 2$, where the emission is dominated by Fe XVIII and Fe XIX. For $\log \xi>2.5$ the overall line emission decreases rapidly as the iron ions become more ionized. This clearly resembles the general trend exhibit by the equivalent widths derived from our models, as is shown in Figure 3 , It is worthwhile to mention that the equivalent widths will increase again for $\log \xi<1$, as can be seen in Figure 7 of Kallman et al. (2004), but those values of the ionization parameter are out of the range of the calculations presented here. The sensitivity of the iron $\mathrm{K} \alpha$ equivalent width to the ionization parameter represents an important potential diagnostic of photoionized plasmas, given its ubiquity in the observed spectra from accreting sources.

Another important diagnostic can be achieved through the dependence of the equivalent width of the $\mathrm{K} \alpha$ line of iron on its abundance with respect to the solar value. This can be seen in Figure 4, where the equivalent width resulting from models with different iron abundances is plotted. In the Figure, connecting lines correspond to a particular ionization parameter (shown next to each curve). For each value of $\xi$, three reflected spectra were calculated assuming $0.2,1$ and 10 times the solar abundance of iron. The resulting equivalent widths are shown as filled circles for each case. It is clear from Figure 4 that the dependence of the $\mathrm{Fe} \mathrm{K} \alpha$ equivalent width on the ionization parameter is almost the same for different abundances of iron, i.e., the shape of the curve in Figure 3 will remain unchanged if we use models with iron over or under abundant, and only the actual values of the equivalent widths will show a variation. This is a consequence of the fact that the Fe K $\alpha$ equivalent width grows linearly with the iron abundance, which is also evident from the Figure. To illustrate this, we include the function $y(x)=10 x$ with a dashed line in Figure 4, which shows a slope similar to all the other curves. 


\subsection{Simulated observations}

The analysis presented previously provide important tools for the interpretation of the X-ray spectra from accreting sources by the use of simple quantities that can be easily derived from the observed spectrum. Nevertheless, the models computed here do not necessarily represent real observed data. First, the spectral resolution used in the models ( $R \sim 350$ ), although comparable to grating spectra, is far superior to what is achieved in CCD observations. Second, the models do not suffer from loss of information due to the natural restrictions involved in real observations, such as the instrument effective area which affects the total number of photons collected. Finally, a real observation is, in general, a mixture of several components and not exclusively represented by a reflected spectrum. In accreting sources such as active galactic nuclei (AGN) and galactic black holes (GBHs), an important component is the contribution of the source of X-rays that illuminates the accretion disk. In fact, it is not clear which fraction of the direct over the reflected component can be present in a given observation, and this proportion is most likely to depend on the geometry and orientation of the system.

In order to account for some of these effects, we have produced simulated CCD observations using our models as the source of the reflected spectra. We have used the fake

it task in XSPEC in combination with the FI XIS response matrices from Suzaku to simulate

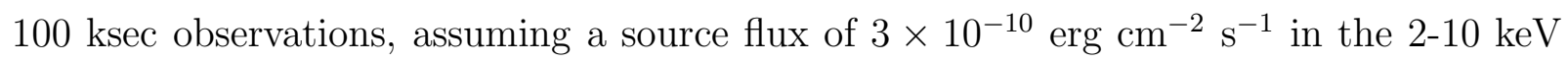
energy band. This flux corresponds to $10 \mathrm{mCrab}$, which is a typical value for bright AGN. We then try to fit the resulting simulated observation with a simple phenomenological model, i.e., the basic continuum is fitted with a power law, and all the relevant features with Gaussians. An example of such a fit is shown in Figure 5. In the upper panel, data points with error bars represent the simulated data using a reflection model with $\log \xi=0.8$, while the solid line shows the best-fit. The lower panel shows the theoretical model used 
with all its components. The continuum is fitted with a power law (thick dashed line), and several emission profiles with Gaussians (thin dotted lines) centered at 2.39, 3.06, 3.8, 6.38 and $7.03 \mathrm{keV}$, corresponding to emission from S VI-X, Ar VIII-XI, Ca X, Fe X-XII K $\alpha$ and Fe K $\beta$, respectively. Finally, a very broad $(\sigma=1.54 \mathrm{keV})$ Gaussian profile centered at $6.1 \mathrm{keV}$ was included in order to properly fit the continuum at lower energies. Clearly, a simple power law cannot describe the continuum for models with low ionization, given the significant modification of the original power law continuum due to the large values of the photoelectric opacity. This very broad curving continuum is required to fit the reflected spectra with $\log \xi \sim 1.1$ or lower. Therefore, this particular profile could be used as a signature of strong reflection from low ionized atmospheres.

We have then produced simulated observations using all the models considered previously, i.e., using the 10 reflected spectra calculated for $\log \xi=0.8,1.1,1.5,1.8, \ldots, 3.8$. By including the Suzaku response matrices we can simulate the effects of the effective area from the instrument, its sensitivity and energy resolution. However, many X-ray observations from accreting sources reveal that the direct, unprocessed radiation as a power-law is detected simultaneously along with the reflected component. Therefore, we have produced additional simulated observations with a source spectrum resulting from the combination of both the reflected and the illuminating components. In order to quantify the proportion of one component to the other we use a $\beta$ parameter, defined as the ratio of the incident over the reflected flux,

$$
\beta=\frac{F_{\text {inc }}}{F_{\text {ref }}}
$$

where both fluxes are defined in the 2-10 keV energy range. Note that this quantity differs from the reflection fraction $R=\Omega / 2 \pi$, where $\Omega$ is the solid angle sustained by the reflector. The relation between the reflection fraction and our $\beta$ parameter is roughly $R \approx 1 / \beta$; such that a pure reflection case corresponds to $\beta=0$ and $R \gg 1$, while in the case of no reflection $\beta \gg 1$ and $R=0$. Because our models do not carry any information about the 
geometry of the reflector, it is not appropriate to derive a reflection fraction $R$ for them. Instead, we use the $\beta$ parameter to quantify the dilution of the spectral profiles by the direct power-law spectrum, in order to compare derived spectral quantities with the those from real observations.

Figures [6, 7 and 8 show the simulated CCD observations using our models with $\log \xi=0.8,1.8$ and 2.8 , respectively. In each figure, the data points in the upper panel show the simulated data for three different values of the $\beta$ parameter, namely, from bottom to top, $\beta=0,0.5$ and 2 . The solid lines are the best-fit for each case, using the same kind of simple models described before. The dots in the lower panel represent the residuals in units of $\sigma$ for all the fits. These figures show the effect of the instrument on the observed spectra. In particular, by comparing the curve for $\beta=0$ in Figure 6 with the corresponding model in Figure 1, it is clear how the narrow Fe $\mathrm{K} \alpha$ and $\mathrm{K} \beta$ lines in the $\log \xi=0.8$ model become much broader features in the simulated observation. As mentioned before, emission from S, $\mathrm{Ar}$ and $\mathrm{Ca}$ ions are the most prominent features at energies $<6 \mathrm{keV}$. It is also important to notice from this Figure the dilution of the spectral profiles due to the inclusion of the direct power-law spectra (i.e., the curves for $\beta=0.5$ and $\beta=2$ ). The iron $\mathrm{K} \alpha$ at $6.4 \mathrm{keV}$ and the sulfur blend at $\sim 2.39 \mathrm{keV}$ emission profiles are clearly detectable, even for $\beta=2$ (i.e., twice as much direct flux as the reflected flux in the $2-10 \mathrm{keV}$ band). However, the weaker emission lines become almost undetectable for $\beta \geq 2$. Most important, not only the atomic features are modified but the continuum as well. The broad Gaussian profile required to fit the continuum in combination with the power-law is no longer required for $\beta=0.5$ or greater. This is important, since this means that this feature is characteristic of low ionization, but also appears exclusively for the pure reflection cases $(\beta=0)$.

Figures 7 and 8 show essentially the same situation. In Figure 7, which corresponds to the simulation generated with $\log \xi=1.8$, it is important to notice the presence of the 
$\mathrm{S}$ XV K $\alpha$ emission line at $\sim 2.45 \mathrm{keV}$, and the $\mathrm{S}$ XVI $\mathrm{K} \alpha$ plus the radiative recombination continua $(\mathrm{RRC})$ at $\sim 2.59 \mathrm{keV}$, which are detectable even in the $\beta=2$ simulated spectrum. The iron $\mathrm{K} \beta$ emission line becomes undetectable for $\beta>1$. Figure 8 shows the $\log \xi=2.8$ simulated spectra. In this case, the iron emission is the only clear and strong feature. However, the iron K-shell emission can no longer be well represented by a single Gaussian profile. Instead, a broad $(\sigma=1.26 \mathrm{keV})$ Gaussian centered at $\sim 6.5 \mathrm{keV}$ accounts for the Comptonized profile, while a narrow one centered at $\sim 6.6 \mathrm{keV}$ accounts for the discrete emission lines that can be seen in the original spectrum of Figure 1, The iron emission can be well fitted even in the $\beta=2$ case, but not for cases with a larger contribution of the direct power-law spectrum.

In Table 1 we show a compilation of the strongest features detectable in the simulated CCD spectra for the pure reflection case $(\beta=0)$. We show the line centroid energy and the equivalent with for each feature. Three of the features are blends of several components, such as the Si VI-X around $2.39 \mathrm{keV}$, the blend of the $\mathrm{S}$ XI $\mathrm{K} \alpha$ line and the Si XIV RRC around $2.59 \mathrm{keV}$, i and the Ar VIII-XI blend around $3.3 \mathrm{keV}$.

\subsection{Comparison with observations}

In order to test the validity of the results obtained with the simulated CCD spectra, we have compared our estimates with those derived from real observations. To do this we have concentrated our attention on the equivalent widths and centroid line energies of the iron $\mathrm{K} \alpha$ emission profile, since this is the strongest emission present in the spectra from most accreting sources. Because the resulting equivalent width is very sensitive to small changes in the fitted continuum, we decided to apply a systematic procedure to calculate the line equivalent width, similar to the one described in Section 3.1. First, we have generated simulated CCD spectra for the 10 different values of the ionization parameter considered in 
this paper $(\log \xi=0.8,1.1,1.5,1.8, \ldots, 3.8)$, and for 5 different values of the $\beta$ parameter from Equation 8 ( $\beta=0,1,2,5,10)$, which covers cases from pure reflection cases up to cases dominated by the direct power-law (no reflection). Then, we fit a simple model to each spectrum, consisting of a power-law for the local continuum and one Gaussian profile for the Fe K-shell emission. The fit is performed in a small energy range concentrated around the iron lines, ignoring any other channels. We choose the range to be 5.7-7.3 keV for spectra with $0.8 \geq \log \xi \geq 2.8$, and $3-10 \mathrm{keV}$ for those with larger ionization parameters, since the Fe K-line is much broader due the Comptonization.

In Figure 9, the line centroid energies and equivalent widths resulting from all our simulated spectra are compared with the same quantities derived from spectra of Seyfert (Sy) galaxies and low mass X-ray binaries (LMXB). In the Figure, filled circles correspond to Sy 1 and 1.2 galaxies, asterisks to Sy 1.5, and filled triangles to Sy 1.8, 1.9 and 2; all taken from the Winter et al. (2009) compilation. Filled squares correspond to the LMXB analyzed by $\mathrm{Ng}$ et al. (2010). The error bars are included as dashed lines. Dots connected with solid lines are the values predicted by our reflection models. Each line corresponds to one particular ratio of the direct over the reflected component, or $\beta$ parameter. From top to bottom, each curve corresponds to $\beta=0,1,2,5$ and 10. Error bars are also included as solid lines. Along each line, the points from left to right correspond to the models with increasing values of the ionization parameter $\xi$.

There are important pieces of information contained in Figure 9. The observed data shows the clear distinction between Sy galaxies and LMXB with respect to their iron emission. Sy galaxies seem to only show the cold iron line at $\sim 6.4 \mathrm{keV}$, while in the LMXB spectra the hot line around $6.7 \mathrm{keV}$ is detected. This yields a marked difference in the ionization of the emitting gas between these two types of sources. According to our models, the cold region of the plot where Sy galaxies appear (for line energies lower than 
$6.5 \mathrm{keV}$ ), correspond to values of $\log \xi<2$, while the hot region (line energies larger than $6.5 \mathrm{keV}$ ), where LMXB show up, covers larger values of ionization, up to $\log \xi=4$. In the cold region, Sy galaxies equivalent widths cover a large range, from 10 to $1000 \mathrm{eV}$. Despite the large error bars in many of the values, equivalent widths larger than $200 \mathrm{eV}$ seem to correspond almost exclusively to Sy 1.5-2 galaxies. Our models predict that the largest values correspond to reflection dominated cases, with $\beta=0,1$ and 2 . Lower equivalent widths (less than $200 \mathrm{keV}$ ), seem to be common to all types of Sy galaxies. However, our models require significant contribution from the direct power-law illuminating spectra to dilute the line profiles and reproduce the lower values of the equivalent widths. Accordingly, we need to produce models with $\beta=10$ to explain the equivalent widths lower than $100 \mathrm{eV}$ (i.e., 10 times more direct power-law flux than the reflected one, or $R \rightarrow 0$ ). Also, it is important to notice that for the lower ionization models the resulting line energies are much closer to each other than for the high ionization ones. At the same time, the data for Seyfert galaxies in Figure 9 shows a large concentration of points at $6.4 \mathrm{keV}$. This is probably due to the fact that sometimes observers fix the line centroid energy to a known value given the poor statistics of the data. This imposes difficulties in the estimation of the ionization parameter when comparing the data values to the ones predicted by the models. Therefore, accurate line energies are needed from the observations in order to improve the diagnostics.

The LMXB observations are more scattered in the hot region of the Figure 9 (line energies larger than $6.5 \mathrm{keV}$ ). Both the models the the observed data predict equivalent widths as small as $\sim 10 \mathrm{eV}$, but no larger than $\sim 200 \mathrm{eV}$. The error bars in the values resulting from the models are much larger than in the low ionization cases, mainly because the line feature is weaker as the ionization increases, since the emission is mostly due to $\mathrm{H}-$ and He-like iron. The line profile is even weaker as the $\beta$ parameter increases, due to the direct power-law dilution. Therefore, in the cases for $\beta=1$ and 2 (third and fourth curves from top to bottom), the line cannot be detected in the spectra simulated with 
highest ionization parameter $(\log \xi=3.8)$. The same occurs for the simulated spectra with $\log \xi=3.5$ in the bottom curve $(\beta=10)$. The ranges of the ionization and the $\beta$ parameters covered in this paper are sufficient to well represent the observed data for both Seyfert galaxies and LMXB.

The fact that the largest equivalent widths predicted by our reflection dominated models correspond mostly to the values found in Sy 1.5-2 galaxies agrees with the general picture that in these sources the direct component of X-ray radiation is being obscured, and only the reflected or reprocessed radiation is observed. Following this idea, we repeated the comparison of our models with a larger and more recent compilation of AGN Seyfert galaxies done by Fukazawa et al. (2010). These authors analyzed high-quality Suzaku data for 88 Seyfert galaxies, and derived line energies, equivalent widths, and hydrogen column densities among other quantities. In Figure 10 we show the equivalent width against the Fe K $\alpha$ line energy derived from these observations. For comparison we use the same scale used in Figure 9, but in this case we use different symbols to show different values of the hydrogen column density $N_{\mathrm{H}}$. In the plot, filled circles represent galaxies with $\log N_{\mathrm{H}}<21 \mathrm{~cm}^{-2}$, asterisks are galaxies with $21 \leq N_{\mathrm{H}}<23 \mathrm{~cm}^{-2}$, and filled triangles are those with $\log N_{\mathrm{H}} \geq 23 \mathrm{~cm}^{-2}$. As in Figure 9, filled squares are the values for the LMXB from $\mathrm{Ng}$ et al. (2010), and dots connected with solid lines are the values predicted by our models. This particular sample has the advantage of being more accurate (lower error bars), and is more consistent since all the data was obtained with the same instrument and analysis technique. However, the information derived from this comparison is consistent with the Winter et al. (2009) compilation. Equivalent widths larger than $200 \mathrm{eV}$ are only observed in sources with large hydrogen column densities $\left(\log N_{\mathrm{H}} \geq 23 \mathrm{~cm}^{-2}\right.$ ), which corresponds to the reflection-dominated models $(0 \leq \beta \leq 2)$. Lower equivalent widths are observed in sources with all values of $N_{\mathrm{H}}$. The majority of sources with low hydrogen column densities (filled circles) have equivalent widths lower than $100 \mathrm{eV}$, consistent with 
models with large contribution of the direct power-law $(\beta \sim 10)$. The agreement between these observations and our models is in general very good. However, there are at least five galaxies with equivalent widths larger than the maximum values predicted by our models $(\sim 1 \mathrm{keV})$ with solar abundances. However, larger equivalent widths can be easily achieved by increasing the iron abundance, as shown in Figure 4 .

\section{Conclusions}

In this paper we have presented a comprehensive study of the emission spectra from accreting sources. Using our new reflection modeling code (García \& Kallman 2010), we have computed the reflected spectra from an X-ray illuminated accretion disk. We have concentrated our analysis to the iron K-shell emission lines, although other spectral features are also discussed. Our models predict equivalent widths for the Fe K $\alpha$ emission of $\sim 10 \mathrm{eV}$ for high ionization parameters ( $\log \xi \sim 4$ ), and maximum values of $\sim 800 \mathrm{eV}$ for models with $\log \xi \sim 1.5$. For lower values of the ionization parameter the equivalent widths decrease to a minimum near $\log \xi \sim 1$, contrary to what other models predict. These differences are due to the atomic data used by each simulation. We have shown that the behavior of the Fe $K \alpha$ equivalent widths with respect to the ionization parameter of the gas is consistent with

the line emissivities shown by Kallman et al. (2004), where the same atomic data was used. Additionally, these equivalent widths display a linear dependency with the iron abundance normalized to its solar values. This seems to be true for all the models within the range of ionization parameter values considered in this paper.

Simple analysis of simulated CCD spectra reveals that for low ionized, reflection dominated cases the $2-10 \mathrm{keV}$ continuum cannot be represented by a simple power-law. Instead, a broad Gaussian profile is also required. This type of continuum can be used as a strong reflection signature. These simulations also indicate that in addition to the iron 
K-shell lines, the $\mathrm{S} x \mathrm{KV} \alpha$ emission line at $2.45 \mathrm{keV}$ is one of the most prominent features in the spectra. For cases with $\log \xi \sim 2$, this line blends with the He-like silicon RRC providing a broad feature that cannot be represented by simple Gaussian profiles. This could also be used as another reflection signature while analyzing observations.

The iron $\mathrm{K} \alpha$ equivalent widths and line centroid energies are in good agreement with values reported in the literature for both AGN Seyfert galaxies, and galactic sources such as LMXB. In particular, both model and observations show that large equivalent widths $(>200 \mathrm{eV})$ can be achieved in many situations. According to our models, the largest values of the equivalent widths correspond to the lower $\beta$ parameters, i.e., reflection dominated cases. Only observational data from AGN Sy 1.8-2 galaxies, or those with $\log N_{\mathrm{H}} \geq 23 \mathrm{~cm}^{-2}$ coincide with these large equivalent widths, but only for low values of the ionization parameter $(\log \xi<2)$. The majority of the Sy 1-1.2, or those sources with $\log N_{\mathrm{H}}<21 \mathrm{~cm}^{-2}$ show equivalent widths below $200 \mathrm{eV}$. In order to reproduce those values with our models, we need to increase the contribution of the direct power-law up to ten times the contribution of the reflected component. These results agree with the general idea that type 1 and 2 Seyfert galaxies only differ on their orientation with respect to the observer. In the case of LMXB, both the model and the observed data coincide in equivalent widths of $\sim 10-200 \mathrm{eV}$. Since all these sources show hotter Fe K-lines (with energies around $6.7-6.9 \mathrm{keV}$ ), they correspond to models with values of the ionization parameter larger than those for $\operatorname{AGN}(2<\log \xi<4)$.

This work was supported by a grant from the NASA astrophysics theory program 05-ATP05-18. This research has made use of NASA's Astrophysics Data System. 
Table 1. Strongest features in the simulated CCD spectra

\begin{tabular}{|c|c|c|c|c|c|c|c|c|c|c|c|}
\hline $\log \xi$ & $(\mathrm{keV})$ & 0.8 & 1.1 & 1.5 & 1.8 & 2.1 & 2.5 & 2.8 & 3.1 & 3.5 & 3.8 \\
\hline \multirow[t]{2}{*}{ Si XIV K $\alpha$} & $E_{\text {line }}$ & & & & 1.98 & 1.93 & 2.01 & 2.01 & & & \\
\hline & EW & & & & 0.19 & 0.26 & 0.04 & 0.02 & & & \\
\hline \multirow[t]{2}{*}{ Si VI-X } & $E_{\text {line }}$ & 2.39 & 2.39 & & & & & & & & \\
\hline & EW & 6.12 & 1.03 & & & & & & & & \\
\hline $\mathrm{S} \times \mathrm{XV} \alpha$ & $E_{\text {line }}$ & & & 2.46 & 2.45 & 2.45 & 2.44 & & & & \\
\hline + Si XIII RRC & EW & & & 0.36 & 0.29 & 0.17 & 0.03 & & & & \\
\hline $\mathrm{S}$ XI $\mathrm{K} \alpha$ & $E_{\text {line }}$ & & & 2.58 & 2.59 & 2.59 & 2.59 & 2.57 & & & \\
\hline + Si XIV RRC & EW & & & 0.05 & 0.09 & 0.10 & 0.04 & 0.03 & & & \\
\hline \multirow[t]{2}{*}{ Ar VIII-XI } & $E_{\text {line }}$ & 3.06 & 3.07 & 3.29 & 3.29 & 3.34 & & & & & \\
\hline & EW & 0.63 & 0.10 & 0.07 & 0.11 & 0.23 & & & & & \\
\hline \multirow[t]{2}{*}{$\mathrm{Ca} \mathrm{X}$} & $E_{\text {line }}$ & 3.81 & 3.83 & 3.84 & & & & & & & \\
\hline & EW & 0.43 & 0.14 & 0.03 & & & & & & & \\
\hline \multirow[t]{2}{*}{$\mathrm{Fe} \mathrm{K} \alpha$} & $E_{\text {line }}$ & 6.38 & 6.38 & 6.39 & 6.41 & 6.46 & 6.61 & 6.61 & 6.65 & 6.74 & 6.92 \\
\hline & EW & 0.38 & 0.84 & 1.07 & 0.87 & 0.65 & 0.42 & 0.37 & 0.39 & 0.16 & 0.04 \\
\hline $\mathrm{Fe} \mathrm{K} \beta$ & $E_{\text {line }}$ & 7.03 & 7.01 & 6.96 & 6.97 & 6.97 & & & & & \\
\hline
\end{tabular}




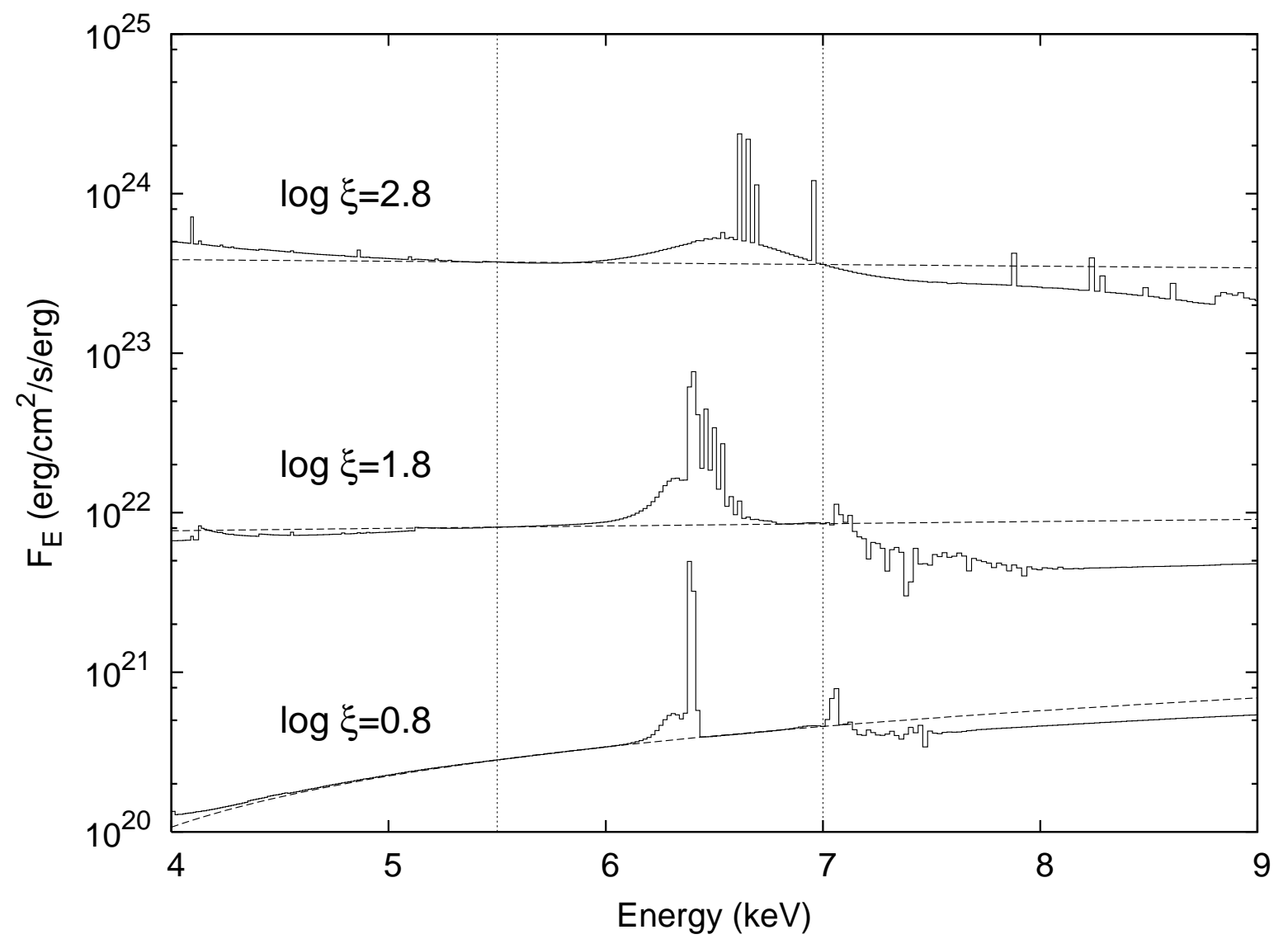

Fig. 1. - Solid curves: emergent spectra from the surface of an accretion disk with constant density $\left(n=10^{15} \mathrm{~cm}^{-3}\right)$ calculated with our reflection code XILLVER for three different illuminations. The log of the ionization parameter is shown next to each curve. The curves are rescaled for clarity. Dashed lines: local continuum used in the calculation of the equivalent widths. Dotted vertical lines are placed at 5.5 and $7 \mathrm{keV}$. 


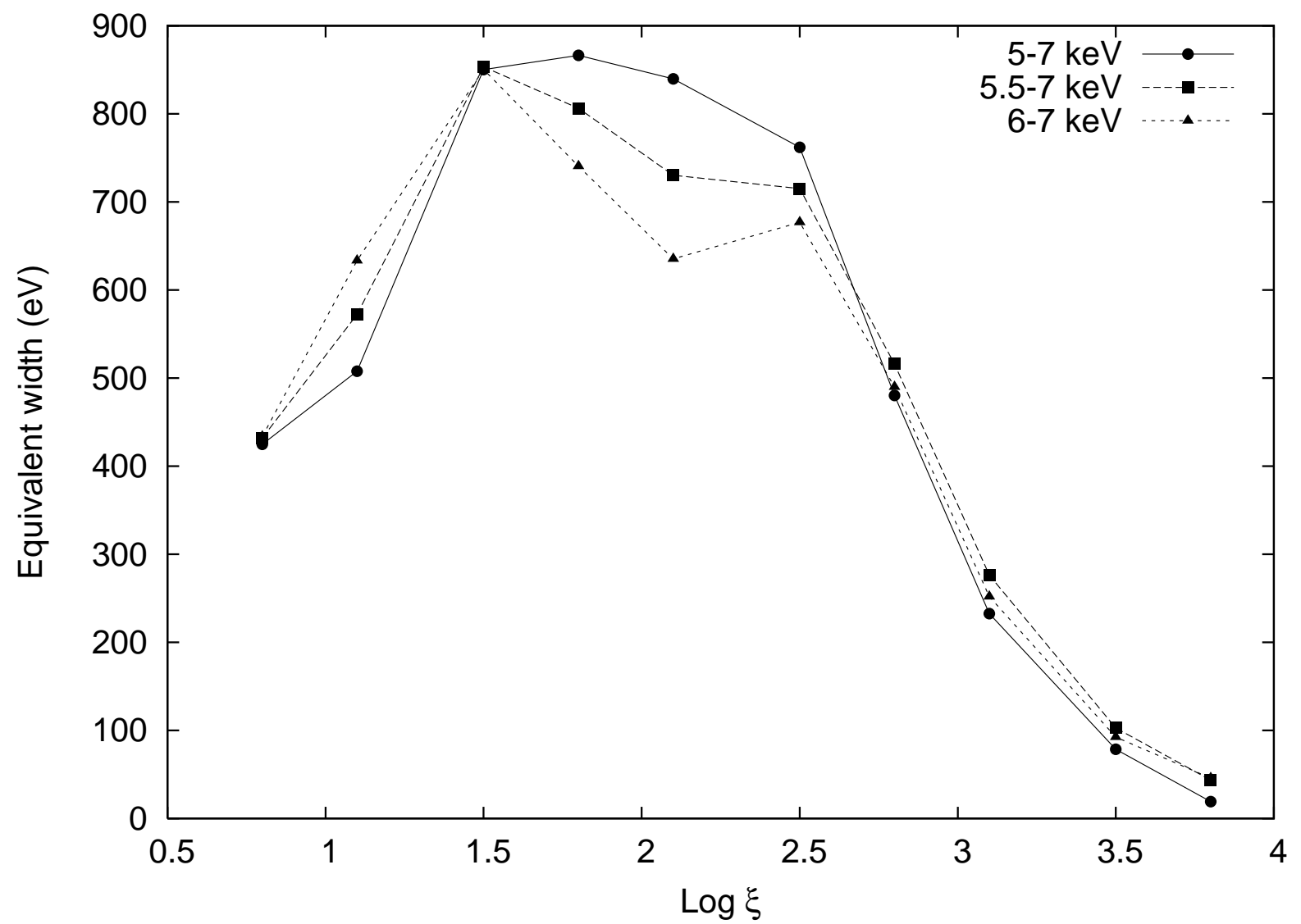

Fig. 2.- Equivalent width of the $\mathrm{Fe} \mathrm{K} \alpha$ line as a function of the $\log$ of the ionization parameter, resulting from three different energy ranges of integration. Connected circles, squares and triangles correspond to $E_{l o w}=5,5.5$ and $6 \mathrm{keV}$, respectively. $E_{h i g h}$ is set equal to $7 \mathrm{keV}$ for all the cases. 


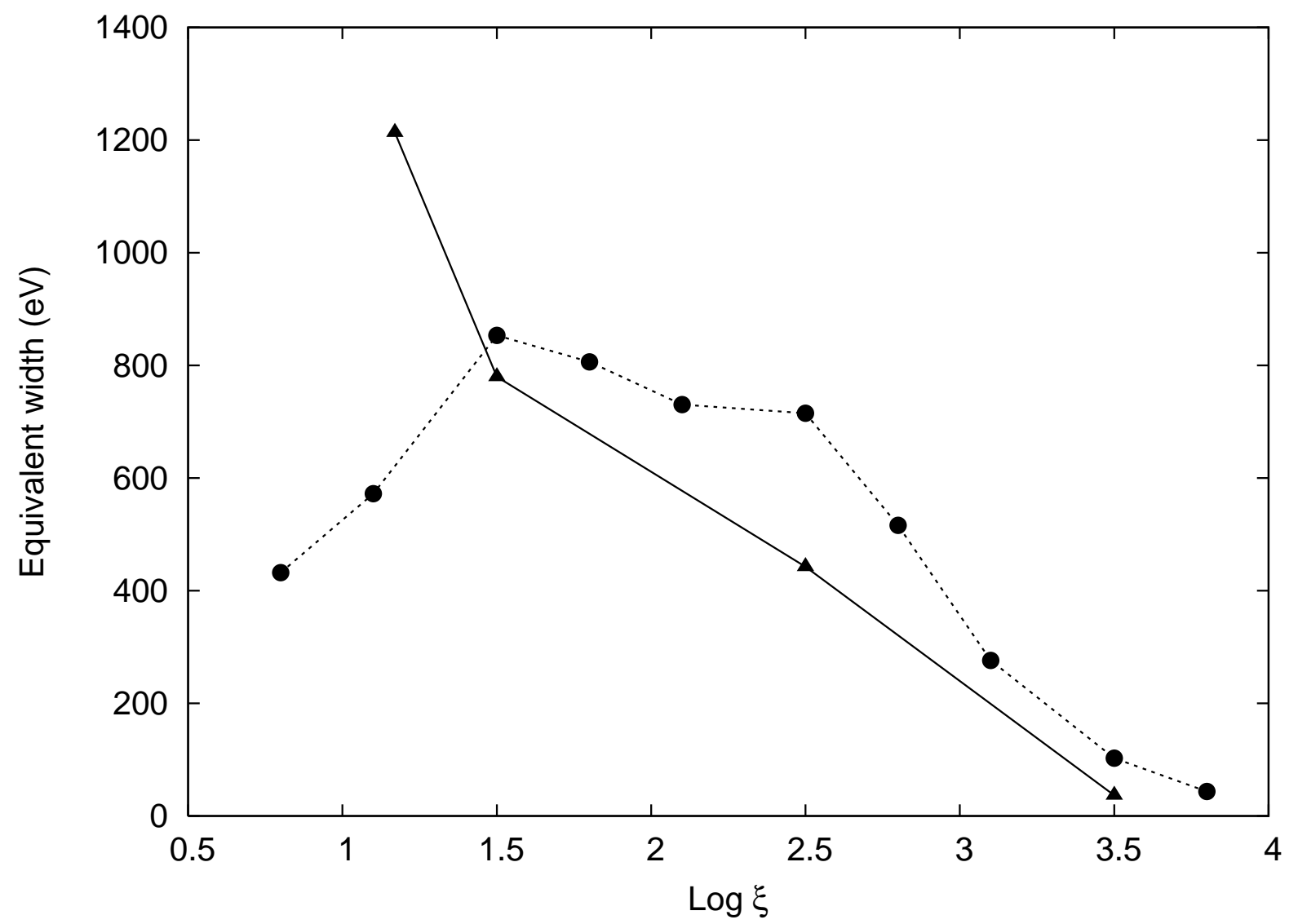

Fig. 3.- Equivalent width of the $\mathrm{Fe} \mathrm{K} \alpha$ line as a function of the $\log$ of the ionization parameter. Connecting circles are the values predicted by our reflection code XILLVER, while triangles correspond to the values predicted by REFLION (Ross \& Fabian 2005). The integration is performed over the 5.5-7 keV energy range in both models. 


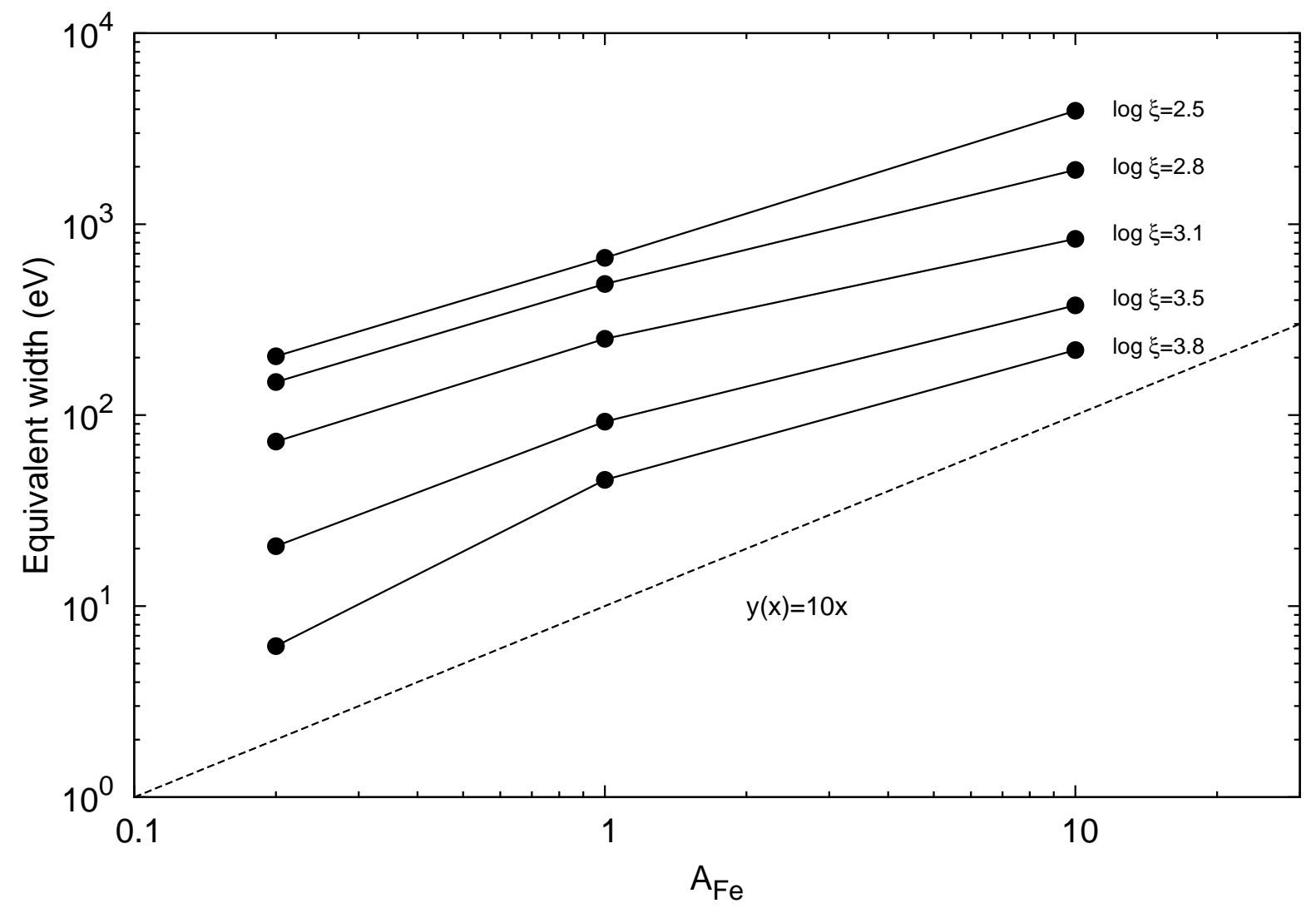

Fig. 4. - Fe K $\alpha$ equivalent width as a function of the iron abundance (with respect to solar). Connecting circles are the values predicted by XILLVER for different illuminations. The value of $\log \xi$ is shown next to each curve. The dashed line represents the function $y(x)=10 x$, to exemplify the linear dependency of these quantities. 


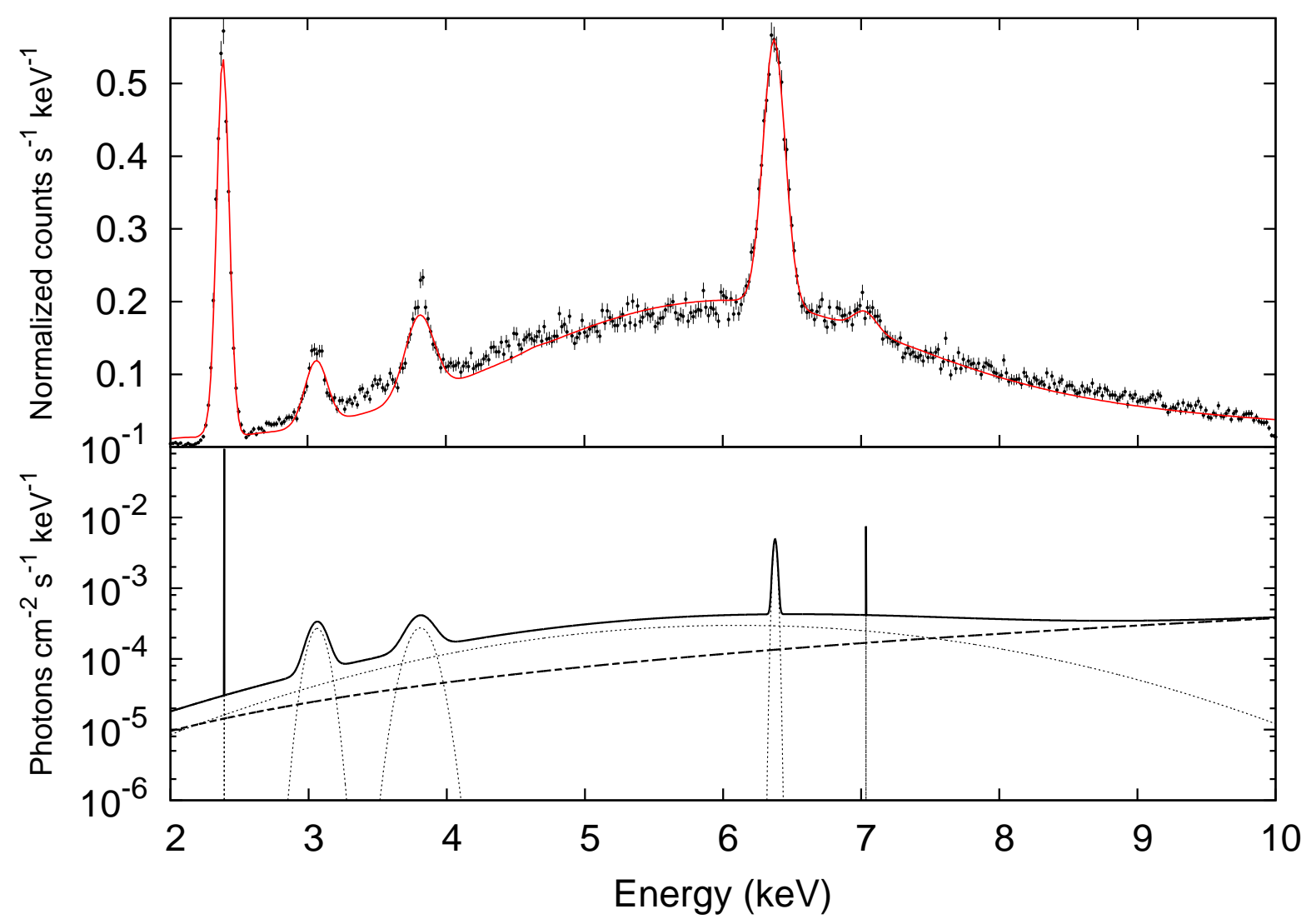

Fig. 5.- Upper panel: data points are the simulated CCD observation produced with our model for $\log \xi=0.8$. The solid line is the best-fit using a simple phenomenological model. Error bars are shown for all the data points. Lower panel: the theoretical model used in the fit is shown with the solid line, while all the individual components are shown with dashed curves for the Gaussian profiles, and with a thick-dashed line for the power-law. 


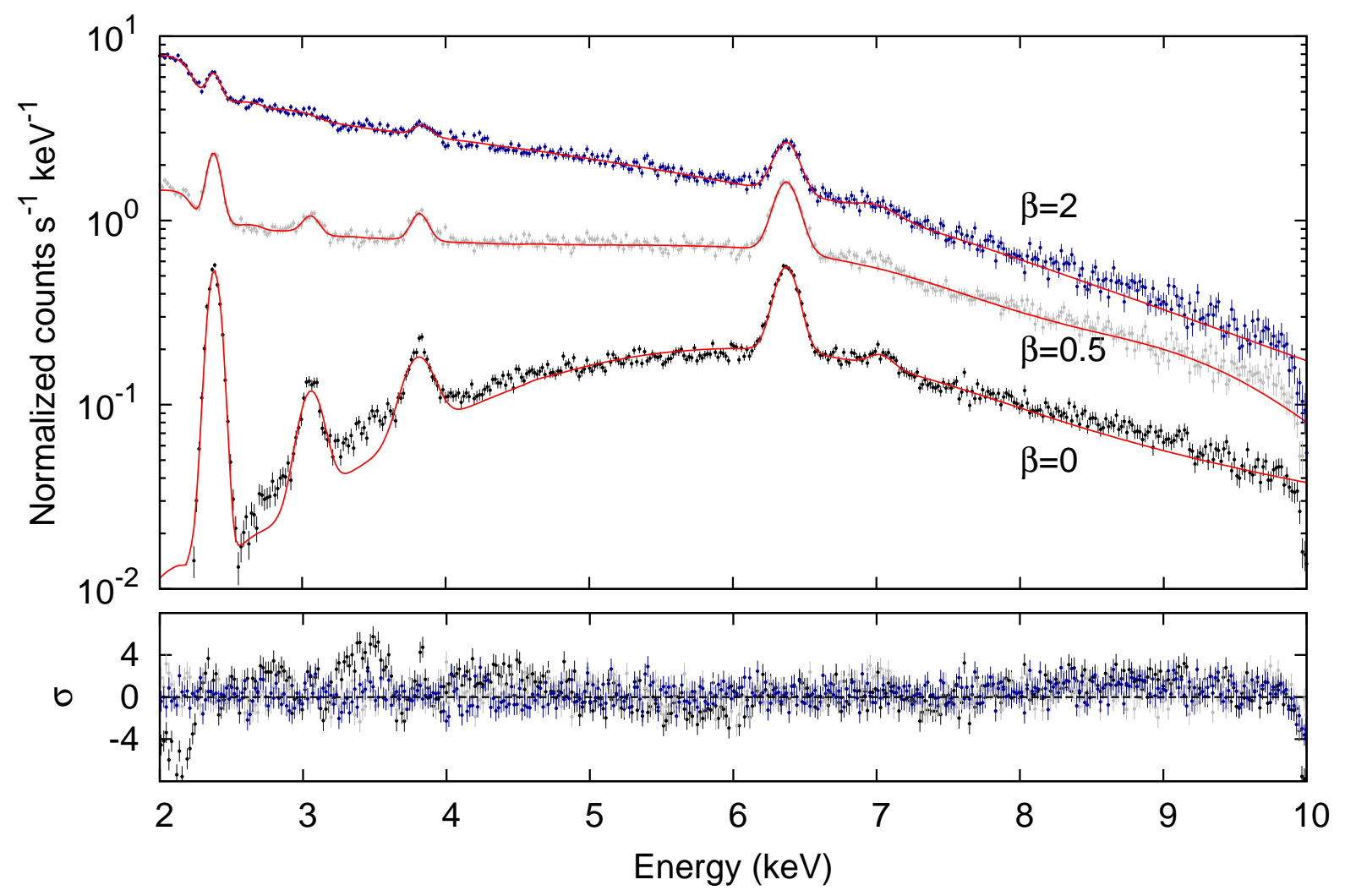

Fig. 6.- Upper panel: data points show the simulated CCD spectra produced with our model for $\log \xi=0.8$, for $\beta=0,0.5$ and 2, from bottom to top. The solid lines are the best-fits for each case. Lower panel: residuals in units of $\sigma$ for all the fits. Error bars are shown for all the data points. 

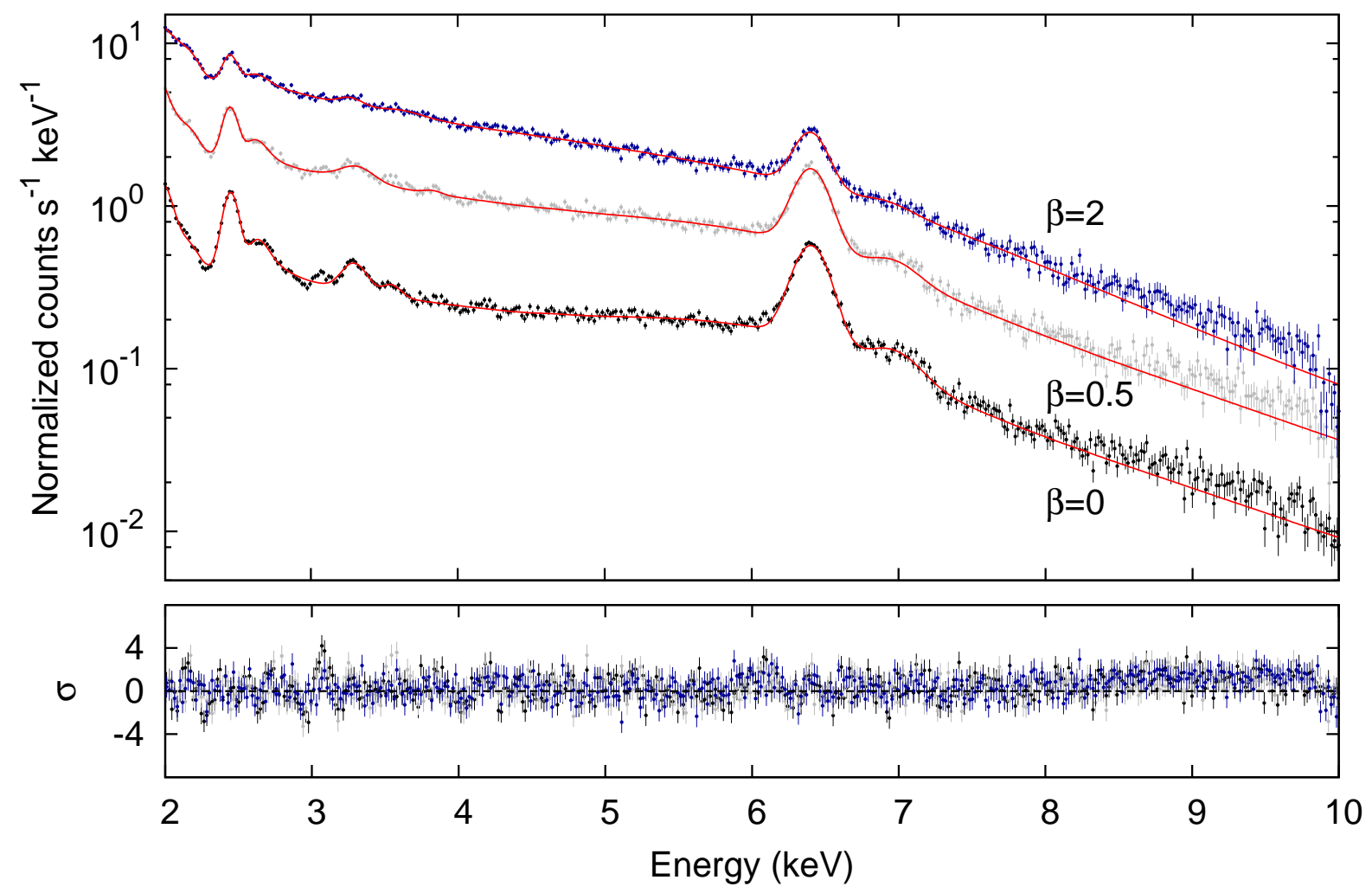

Fig. 7.- Upper panel: data points show the simulated CCD spectra produced with our model for $\log \xi=1.8$, for $\beta=0,0.5$ and 2, from bottom to top. The solid lines are the best-fits for each case. Lower panel: residuals in units of $\sigma$ for all the fits. Error bars are shown for all the data points. 

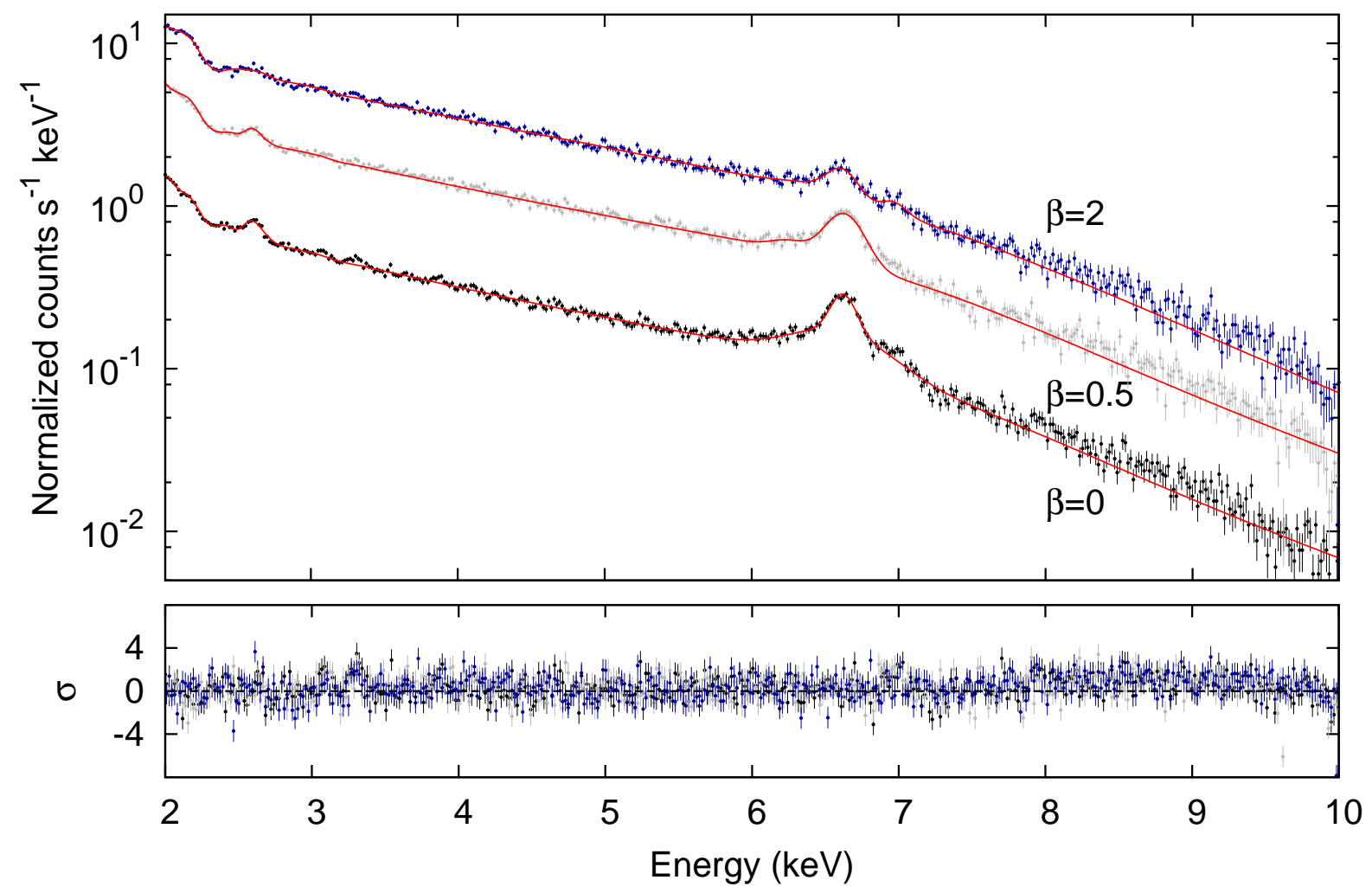

Fig. 8.- Upper panel: data points show the simulated CCD spectra produced with our model for $\log \xi=2.8$, for $\beta=0,0.5$ and 2 , from bottom to top. The solid lines are the best-fits for each case. Lower panel: residuals in units of $\sigma$ for all the fits. Error bars are shown for all the data points. 


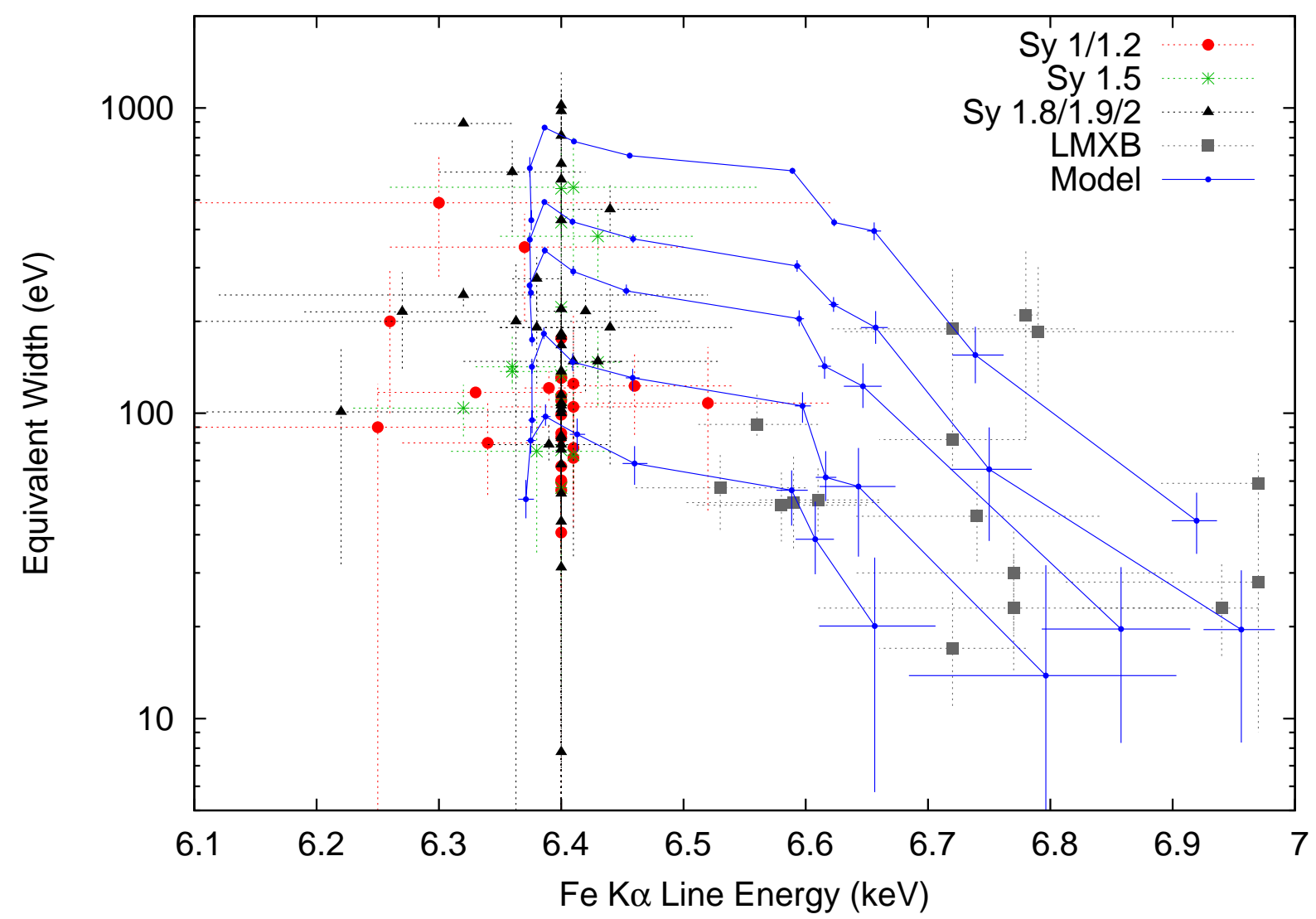

Fig. 9.- Equivalent width versus centroid energy of the Fe K $\alpha$ line. Filled circles, asterisks and filled triangles correspond to AGN Seyfert 1-1.2, 1.5 and 1.8-2 galaxies, respectively (Winter et al. 2009). Filled squares correspond to LMXB (Ng et al. 2010). Dots connected with solid lines are the values predicted by our models with $0.8 \leq \log \xi \leq 3.8$ (from left to right). From top to bottom, each curve corresponds to $\beta=0,1,2,5$ and 10. Error bars are shown for both data and model points. 


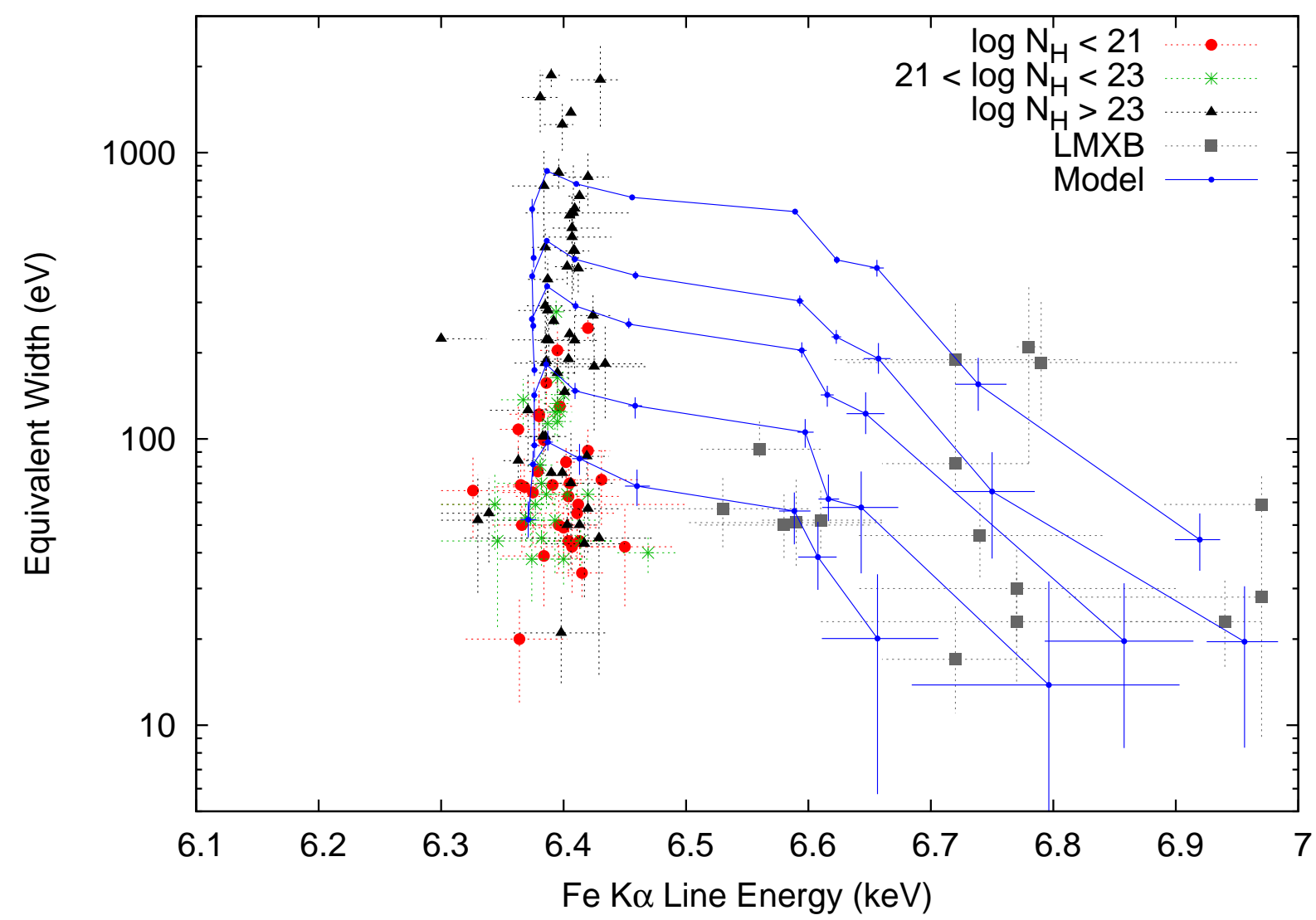

Fig. 10.- Equivalent width versus centroid energy of the Fe K $\alpha$ line. Filled circles, asterisks and filled triangles correspond to AGN Seyfert galaxies with $\log N_{\mathrm{H}}<21,21<\log N_{\mathrm{H}}<23$, and $\log N_{\mathrm{H}}>23 \mathrm{~cm}^{-2}$, respectively (Fukazawa et al. 2010). Filled squares correspond to LMXB (Ng et al. 2010). Dots connected with solid lines are the values predicted by our models with $0.8 \leq \log \xi \leq 3.8$ (from left to right). From top to bottom, each curve corresponds to $\beta=0,1,2,5$ and 10 . Error bars are shown for both data and model points. 


\section{REFERENCES}

Ballantyne, D. R., Ross, R. R., \& Fabian, A. C. 2001, MNRAS, 327, 10

Bautista, M. A., \& Kallman, T. R. 2001, ApJS, 134, 139

Blackman, E. G. 1999, MNRAS, 306, L25

Cunto, W., Mendoza, C., Ochsenbein, F., \& Zeippen, C. J. 1993, A\&A, 275, L5+

Czerny, B., \& Zycki, P. T. 1994, ApJ, 431, L5

Done, C., Mulchaey, J. S., Mushotzky, R. F., \& Arnaud, K. A. 1992, ApJ, 395, 275

Dumont, A.-M., Czerny, B., Collin, S., \& Zycki, P. T. 2002, A\&A, 387, 63

Fukazawa, Y., et al. 2010, ArXiv e-prints

García, J., \& Kallman, T. R. 2010, ApJ, 718, 695

García, J., Mendoza, C., Bautista, M. A., Gorczyca, T. W., Kallman, T. R., \& Palmeri, P. 2005, ApJS, 158, 68

García, J., et al. 2009, ApJS, 185, 477

Hummer, D. G., Berrington, K. A., Eissner, W., Pradhan, A. K., Saraph, H. E., \& Tully, J. A. 1993, A\&A, 279, 298

Kallman, T., \& Bautista, M. 2001, ApJS, 133, 221

Kallman, T. R., Palmeri, P., Bautista, M. A., Mendoza, C., \& Krolik, J. H. 2004, ApJS, 155,675

Krolik, J. H., Madau, P., \& Zycki, P. T. 1994, ApJ, 420, L57

Landi, E., \& Phillips, K. J. H. 2006, ApJS, 166, 421 
Magdziarz, P., \& Zdziarski, A. A. 1995, MNRAS, 273, 837

Matt, G., Fabian, A. C., \& Ross, R. R. 1993, MNRAS, 262, 179

-. 1996, MNRAS, 278, 1111

Miller, J. M. 2007, ARA\&A, 45, 441

Nandra, K., \& Pounds, K. A. 1994, MNRAS, 268, 405

Nayakshin, S., \& Kallman, T. R. 2001, ApJ, 546, 406

Nayakshin, S., Kazanas, D., \& Kallman, T. R. 2000, ApJ, 537, 833

Ng, C., Diaz Trigo, M., Cadolle Bel, M., \& Migliari, S. 2010, ArXiv e-prints

Palmeri, P., Quinet, P., Mendoza, C., Bautista, M. A., García, J., \& Kallman, T. R. 2008, ApJS, 177, 408

-. 2010, A\&A, 177, 408

Pounds, K. A., Nandra, K., Stewart, G. C., George, I. M., \& Fabian, A. C. 1990, Nature, 344,132

Poutanen, J., Nagendra, K. N., \& Svensson, R. 1996, MNRAS, 283, 892

Ralchenko, Y., Kramida, A. E., Reader, J., \& NIST ADS Team. 2008, NIST Atomic Spectra Database, version 3.1.5 (Gaithersburg: NIST), http://physics.nist.gov/asd3

Ross, R. R., \& Fabian, A. C. 1993, MNRAS, 261, 74

-. 2005, MNRAS, 358, 211

-. 2007, MNRAS, 381, 1697

Ross, R. R., Fabian, A. C., \& Brandt, W. N. 1996, MNRAS, 278, 1082 
Rozanska, A., \& Czerny, B. 1996, Acta Astron., 46, 233

Shakura, N. I., \& Sunyaev, R. A. 1973, A\&A, 24, 337

Summers, H. P. 2004, The ADAS User Manual, version 2.6, http://adas.phys.strath.ac.uk

Tarter, C. B., Tucker, W. H., \& Salpeter, E. E. 1969, ApJ, 156, 943

Winter, L. M., Mushotzky, R. F., Reynolds, C. S., \& Tueller, J. 2009, ApJ, 690, 1322 